\title{
Idée de "mot juste" et enseignement du vocabulaire au cycle 3 de l'école élémentaire
}

\author{
Florence Charles \\ IUFM de Bretagne, Université de Bretagne occidentale \\ charles.florence@orange.fr \\ «Une intention conceptuelle peut être dite de \\ plusieurs façons, allant du "mot juste" (l'orthonyme) \\ à la périphrase ludique." (...) "L'expression «il \\ faut appeler un chat un chat » révèle cette intuition \\ que les entités ont une désignation privilégiée, \\ immédiate, dans une situation, un environnement \\ bien déterminé. $»$.
}

Bernard Pottier (1992 : 42, 123) par le terme d'orthonyme - le mot le plus adéquat qui est sans signification ajoutée et n'est l'objet d'aucune recherche connotative - fait référence dans sa sémantique générale à propos du choix des signes - ce que l'auteur appelle «l'axe onymique » - à un type de justesse lexicale, que désigne parfois l'appellation de "mot exact" ou bien de "mot propre". L'appellation de "mot juste", qui se présente quant à elle comme une appellation plus générique, susceptible d'englober et de subsumer des cas de figure différents - par exemple le mot "propre", le mot "précis", le mot "approprié" -, fait état d'un point de vue métalinguistique : le locuteur relativement à une situation de communication donnée, qu'il s'agisse à l'oral ou à l'écrit d'une situation de réception ou bien d'une situation de production, juge de la propriété d'un mot ou de mots et exprime un sentiment de convenance. L'appréciation portée qualifie, comme le précise Pottier, une opération langagière de désignation d'entités extralinguistiques qui se réalise dans un acte de nomination. Aussi l'appellation de "mot juste" sur un plan ontologique est-elle liée pour une bonne part à la problématique des mots et des choses, par conséquent à la problématique des relations langue/monde - ce dont le langage et la langue peuvent parler, ce à quoi ils réfèrent. Jacqueline Authier-Revuz (1995 : 548) identifie et décrit au chapitre VII de son étude les formes linguistiques et discursives de dédoublement énonciatif où le locuteur glose la coïncidence ou bien la noncoïncidence des mots et des choses dans l'acte de nomination :

\begin{abstract}
Dans les trois cas, la figure centrale est celle du «mot juste », celui qui fait coïncider le dit au vouloir dire; et qu'il soit représenté comme trouvé (1), poursuivi (2), ou manqué (3), la mise en question locale de cette coïncidence confirme différentiellement le reste de la chaîne comme «allant de soi » quant à cette coïncidence du mot à la chose, c'est-à-dire sans que s'ouvre dans la nomination l'espace de cette interrogation, dont, en y répondant, les formes avouant un défaut, exprimant une incertitude, et, tout autant, affirmant l'adéquation, témoignent, dès lors qu'elles sont explicites.
\end{abstract}

Ce sentiment de coïncidence repose sur la croyance en l'univocité du rapport entre langue et monde, une croyance qui forme une conception très ancienne et une conception très courante de la langue comme miroir du monde (Paveau et Rosier, 2008). Le terme "propre" est celui qui désigne le mieux les êtres ou les choses dans une perspective de correspondance entre la langue et le monde.

Par ce qui la définit l'appellation de "mot juste" suppose donc de la part du locuteur la prise en compte de considérations ou critères qui fondent le sentiment de convenance ressenti et orientent le mouvement de mise en relation dont procède le point de vue métalinguistique exprimé : autrement dit des connaissances et des conceptions sont en jeu, notamment des connaissances lexicales, encyclopédiques et discursives, ainsi que des conceptions relatives en particulier à la langue et au langage. Les différents traits mentionnés qui forment l'assise de l'appellation expliquent le choix que je fais de considérer en tant qu'idée ce qu'implique et ce que signifie l'appellation de "mot juste". C'est pourquoi je préfère parler d'idée de "mot juste" et placer constamment entre guillemets l'appellation. L'un des exemples que donne 
Pottier (1992) peut être tenu pour significatif du caractère de point de vue - au double sens d'opinion et de façon d'appréhender les emplois lexicaux - constitutif de l'idée, partant d'un certain caractère de variabilité : en relation avec une pratique professionnelle et des connaissances scientifiques, le nom céphalée représente pour le médecin, de façon générale s'entend, un orthonyme, alors que pour le malade c'est plutôt le composé mal de tête qui dans un registre de langue plus courant, moins connoté et sans référence particulière à un corps de connaissances constitué, fait figure de désignation immédiate ou de désignation plus immédiate.

L'idée de "mot juste" renvoie par conséquent à une expérience métalinguistique ordinaire et banale : elle se présente dans une certaine mesure comme le fruit d'une expérience langagière "empirique" et se définit comme l'expression d'un sentiment métalinguistique spontané. Le corpus rassemblé par Authier-Revuz (1995) pour ce qui concerne les «boucles méta-énonciatives 》 où l'énonciateur représente explicitement l'association adéquate/inadéquate des mots et des choses dans l'acte de nomination est constitué d'exemples empruntés à des discours sociaux de toutes sortes : conversations privées, presse écrite, publications spécialisées, œuvres littéraires... Authier-Revuz (1995) note que cette famille de boucles s'observe dans le dire en général, dont la parole quotidienne. L'idée de "mot juste" d'après mes investigations est présente également d'une manière manifeste et repérable dans une littérature de recommandations qui dispense des conseils en matière de style. Il en va de même d'une littérature qui, destinée à des scolaires, des étudiants ou bien un public plus large, vise à dispenser des connaissances lexicales, en d'autres termes vise à enrichir ou affiner le vocabulaire de ses lecteurs en faisant mieux connaître à ces derniers le lexique de la langue française et en prodiguant à cette occasion des conseils concernant l'emploi des mots. L'idée dans ces discours se teinte de normativité sur le plan à la fois linguistique et langagier. L'idée de "mot juste" peut donc être étudiée en divers lieux ou depuis ces divers lieux.

L'objet de la présente contribution est de communiquer une partie des résultats d'une recherche que j'ai consacrée à l'étude de l'idée en contexte scolaire, celui de l'enseignement du vocabulaire au cycle 3 de l'école élémentaire. Mon propos est de montrer dans quelle mesure, c'est-à-dire en quoi et pourquoi, l'idée de "mot juste" est opératoire en ce contexte : en quel sens elle sous-tend et modèle des conceptions qui peuvent présider à de possibles choix didactiques. Mon hypothèse est que l'idée de "mot juste" représente une possible clé de compréhension permettant à la fois de rendre compte de logiques conceptuelles qui sont à l'œuvre dans l'enseignement du vocabulaire, d'éclairer des dynamiques et d'interroger des aspects plus problématiques. C'est ainsi que dans le cadre de cet article j'expliciterai en premier lieu dans quelle mesure l'idée de "mot juste" est active en identifiant les points d'émergence de l'idée. Puis, je déplierai différentes conceptions en jeu concernant les domaines respectifs de la langue, du langage, de l'enseignement et de l'apprentissage ; aussi serai-je amenée à souligner des dynamiques c'està-dire à marquer les liens pouvant relier des conceptions. Dans une troisième et dernière partie, je pourrai alors expliquer dans quelle mesure et donc pourquoi l'idée de "mot juste" d'après les sources étudiées peut être considérée comme un révélateur de lignes de force conceptuelles et de questions que pose l'enseignement du vocabulaire.

\section{Les points d'émergence de l'idée en contexte d'enseignement lexical}

Avant de recenser les différents points d'émergence que j'ai identifiés, je présenterai brièvement les sources prises en compte.

\subsection{Les sources étudiées}

Les sources constituées auxquelles l'analyse fera référence correspondent à des pratiques de classe et à des discours sur ces pratiques. Elles privilégient le point de vue de l'enseignant tel que celui-ci se représente en déclarations et en actes dans les données considérées. Les sources rassemblées sont de différentes natures : un corpus de productions écrites collectées dans trois classes de CM2 (six corps de productions : trois en histoire, trois en littérature) qui ont été corrigées par l'enseignant; des entretiens 
individuels, qui relèvent de la famille des entretiens d'explicitation semi-directifs (années 2005/06 et 2006/07). Au cours de trois de ces entretiens, les professeurs ont décrit dans leurs grandes lignes les deux séquences d'enseignement conduites en histoire et en littérature, des séquences comportant un enseignement lexical explicite, finalisées par la production d'un écrit ; la présentation faite s'est accompagnée d'une remise de documents de classe (documents support, activités menées, listes de mots travaillés, grilles d'auto-évaluation et d'évaluation). Les trois autres entretiens, qui ont porté sur une sélection de trois à quatre productions de la classe tant en histoire qu'en littérature, ont amené les professeurs à préciser leurs normes d'évaluation. Afin de rendre possible une approche comparative des pratiques observées, $j$ 'avais passé une commande auprès des enseignants qui se voulait suffisamment ouverte de telle sorte que ceux-ci puissent effectuer des choix personnels au sein du cadre imposé : un enseignement lexical identifié et explicite (un vocabulaire lié à l'étude du siècle de Louis XIV en histoire; les verbes dits de parole en littérature); la production d'un écrit individuel au libre choix de l'enseignant (nature de la production, genre d'écrit, moment au sein de la séquence d'enseignement).

Voici les consignes d'écriture qui en histoire ont été respectivement données aux élèves dans chacune des classes :

- "Raconte quelle est la situation de la France (vie dans les campagnes et rôle des seigneurs) lorsque Louis XIV prend le pouvoir; Raconte pourquoi Louis XIV a fait construire le château de Versailles en plaçant les mots "Cour », " s'endetter », "pension », " titre de noblesse », " habits luxueux », " mener grand train $》$, et « courtisan » dans ta production »;

- «Je rédige un article qui aurait pu être dans un journal du XVIIème siècle» (l'article qui est fictif relate la disparition du chien/de la chienne du roi/de la reine selon les productions);

- «Utilise les mots suivants pour écrire un texte sur le siècle de Louis XIV : cour, Versailles, monarchie absolue, paysans, nobles, clergé, château, impôts, armée, gabelle, dîme, villes fortifiées, manufactures, protestants, révoquer, édit de Nantes, bourgeois, progrès techniques, famines ».

En littérature, les trois professeurs ont relié un enseignement des verbes dits de parole à l'apprentissage de la production de paroles rapportées, directement ou indirectement. Pour ce qui est de l'écrit à produire, les élèves se sont conformés aux consignes suivantes :

- réécrire à propos d'une version de la fable de La Fontaine Le renard et le bouc un passage de paroles rapportées indirectement en passage de paroles rapportées directement puis inventer une suite et fin possible à la fable ;

- suite à la lecture/étude d'un roman adapté de l'histoire de Robinson Crusoë, relater sous forme d'un dialogue la rencontre sur une île déserte entre un personnage narrateur et un Robinson ;

- transformer en adoptant le point de vue d'un narrateur témoin les dialogues et indications narratives (script) d'une des deux scènes du film Le gendarme se marie - la scène du thé ou bien celle du cours de danse - en un récit comprenant des passages de paroles rapportées indirectement.

\subsection{Les facettes de l'idée de "mot juste"}

Les points d'ancrage de l'idée qui ont été relevés ont trait aux finalités assignées à l'enseignement du vocabulaire, aux normes de l'évaluation et à des objets d'enseignement.

\subsubsection{Les finalités de l'enseignement lexical}

C'est sur le plan des finalités que l'idée de "mot juste" est à l'œuvre de la façon la plus manifeste, autrement dit de façon première. L'enseignement dispensé vise notamment un emploi des mots et en particulier un emploi approprié si bien que les deux orientations majeures que représentent la connaissance et l'emploi des mots s'apparentent aux deux faces d'une même médaille. Les trois enseignants dont des pratiques ont été observées affichent en histoire et en littérature une attente 
d'emploi, autrement dit de réinvestissement du vocabulaire travaillé. L'attente renvoie à une finalité de l'enseignement lexical qui se présente comme principale. En comparaison, la finalité d'emploi approprié ressort moins sur le plan didactique considéré. L'enseignement lexical s'inscrit donc dans une dynamique téléologique et fonctionnelle et prend sens notamment par rapport aux pratiques langagières de production. Il est pensé comme contribuant à la fois à l'exercice, à la construction ou au développement ainsi qu'à la maîtrise de ces pratiques.

La production d'un écrit indépendamment de la commande initiale que j'avais faite tendrait à occuper la première place : chaque professeur a, en effet, choisi de clore la séquence d'enseignement en histoire et en littérature par la production d'un écrit qui représente une évaluation de fin de séquence. La production d'écrit quel que soit son genre d'appartenance fait figure par conséquent de lieu privilégié - le lieu par excellence ? - de l'évaluation des connaissances lexicales. L'enseignement du vocabulaire quant à lui parait être pensé tout particulièrement en littérature dans la perspective d'une mélioration des performances lexicales en production: il vise conjointement une extension du répertoire lexical de l'élève, une diversification de ce répertoire ainsi qu'un développement du lexique actif de l'élève. L'emploi des mots et en l'occurrence un emploi approprié sont perçus comme le signe d'une maîtrise ou du moins d'un cheminement de l'élève vers une maîtrise lexicale et langagière. La production d'un écrit est donc envisagée à la fois comme un temps d'apprentissage (un temps d'emploi des mots), un temps et une voie de l'évaluation des acquisitions lexicales.

En continuité avec les finalités maîtresses de l'emploi et de l'emploi approprié des mots, le champ des normes de l'évaluation se présente comme un autre espace d'émergence de l'idée de "mot juste", un espace qui permet d'approcher la notion d'appropriation et par voie de conséquence des définissants possibles de la justesse lexicale.

\subsubsection{Les normes de l'évaluation lexicale}

L'idée de "mot juste" en correspondance étroite avec la finalité d'emploi des mots se montre active au premier chef à travers la norme générale de l'emploi ou du réinvestissement des mots travaillés. C'est le contexte d'enseignement, en effet, qui confère à ces derniers le statut de "mot juste", des "mots justes" en puissance. Les trois enseignants attendent en littérature dans un récit d'invention comportant des passages de paroles rapportées directement ou indirectement pour l'une des classes l'emploi de verbes de parole qui ont été étudiés ou bien rencontrés au cours de la séquence ; en histoire, deux des professeurs attendent l'emploi d'un vocabulaire imposé qui figure dans la consigne d'écriture sous forme d'une liste et le troisième attend l'emploi d'un vocabulaire identifié, abordé lors de l'étude du siècle de Louis XIV. La norme de l'emploi ou du réinvestissement a d'ailleurs été présentée par deux professeurs lors des entretiens comme une norme première en histoire ("l'aptitude à réinvestir du vocabulaire acquis dans une période »). Parce que la norme établit une sorte de contrat entre l'élève et l'enseignant, elle peut être qualifiée de norme didactique. L'emploi ou le réinvestissement des mots travaillés est donc interprété comme un indice d'acquisition effective ou bien d'acquisition en cours.

Depuis cette ligne directrice de l'emploi ou du réinvestissement, l'idée de "mot juste" est présente de façon plus spécifique et plus identifiée dans la norme de l'appropriation lexicale qui se signale en ces termes ou bien à travers la norme de la précision ou spécificité lexicale. Les deux normes sont à l'œuvre dans l'évaluation lexicale des productions collectées dans les trois classes de CM2. Celle de l'appropriation est explicite : elle est communiquée aux élèves lors de la passation orale des consignes (« utiliser le bon verbe », «employer les verbes à bon escient ») et est signifiée en tant que critère dans les grilles d'évaluation («Les verbes sont bien choisis»; «Le lecteur comprendra bien qu'il s'agit d'un événement du XVIIème siècle (vocabulaire employé, pas d'erreurs historiques...)). Un des trois enseignants a précisé lors d'un entretien que la norme de l'appropriation constituait pour lui tant en littérature qu'en histoire un critère second par rapport au critère du réinvestissement. La norme de la précision ou spécificité lexicale se signale quant à elle d'une façon plus indirecte et plus implicite, tout particulièrement en histoire. C'est en effet le champ disciplinaire qui confère le statut de mot "précis" ou de mot "spécifique" aux mots étudiés en référence à une période historique déterminée. La norme de la 
précision ou spécificité se trouve alors incluse dans celle du réinvestissement et découle d'elle. Aussi dans les données considérées apparait-elle latente et ne se déclare-t-elle pas ouvertement. En littérature, la norme quoique implicite est plus visible. L'attente de l'emploi par l'élève de différents verbes de parole qui ont été travaillés sous-entend l'attente de l'emploi de verbes de parole "précis", plus "précis" que dire ou répondre; l'un des enseignants a systématiquement notifié comme "erreur" en marge de la copie l'emploi du verbe dire. D'autres faits tendent à corroborer la présence et l'efficience de la norme de la précision : un professeur qui dans une fiche outil remise aux élèves donne comme exemples de «verbes plus précis " les verbes répliquer, proposer, s'indigner, suggérer, bredouiller, a qualifié lors d'un entretien de "verbes passe-partout » les verbes dire et répondre; un autre a caractérisé le sémantisme d'un verbe de parole comme « une façon de parler».

L'analyse croisée de l'ensemble des sources - productions écrites, grilles d'évaluation, consignes orales et écrites passées auprès des élèves, déclarations des trois professeurs lors des entretiens - permet de se pencher sur des fondements du sentiment de justesse, autrement dit sur des définissants possibles de la justesse lexicale. Le définissant principal et prégnant est de nature sémantique. Les commentaires faits par les enseignants lors des entretiens, quand il leur a été demandé d'expliciter un critère ( « Les verbes de parole sont bien choisis » : "il n'y a pas de contresens les verbes sont pertinents ») ou d'évaluer sur le plan lexical des passages ou bien l'ensemble d'une production d'élève, confirment ce que laissent pressentir des annotations. En voici deux exemples :

« il [Louis XIV] ne les engage pas il leur verse une pension il [l'élève scripteur] aurait dû mettre titre de pension il s'est trompé de verbe le groupe verbal est utilisé à mauvais escient ", "c'est lui [l'élève] qui interprète il utilise des termes actuels ces mots n'ont pas de sens à l'époque de Louis XIV» («Louis XIV "engage des courtisan" pour lui raconter qu'il est Beau et qu'il est grand et que c'est le plus grand roi de France »; l'enseignant a souligné et mis entre guillemets l'erreur engage des courtisan);

«dans l'histoire elle [le personnage de Josepha] n'a pas raconté il faut replacer dans le contexte elle ne raconte rien du tout ils [les élèves] ont une mauvaise représentation du verbe raconter c'est quand même long dans l'action ils emploient raconte-moi pour dis-moi» ("Josephane chuchote a Cruchot qu'elle âge avait sa fille. Cruchot surpris de cette question affirme qu'elle etait toute petite. Madame La colonel raconta qu'elle aime bien les ptits enfants. »).

Des considérations d'ordre syntagmatique - propriétés syntaxiques des mots et faits de cooccurrence -, bien qu'elles soient présentes dans un ensemble de données, semblent être plus en retrait. Chez l'un des enseignants, un soulignement signale en littérature dans deux copies, ce de façon ponctuelle et non systématique, des erreurs d'ordre syntagmatique : "et le capitaine de bord nous avaient entendus", «mais il eu une tempête, et le bateau chavira et il coula. Je suis le seul naufragé du bateau ", "Une fois le délai fini $»$. Chez les deux autres enseignants, les propos tenus lors d'un entretien inclinent à penser que des considérations d'ordre syntagmatique paraissent être absorbées et donc éclipsées par des considérations d'ordre sémantique :

«elle [le personnage de Josepha] lui a posé une question il faut replacer dans le texte le si devrait lui mettre la puce à l'oreille l'utilisation du si n'est pas pertinente », "il y a un problème au niveau du complément c'était forcément demander cela induit un verbe de questionnement" ("Le François lui demanda s'il avait une fille. Ludovic Cruchot répondit d'un aire doux qu'il avait une fille. Madame Josepha la colonel reprit si elle était avec lui.»);

«l'idée d'intervenir sous-entend une action» («mais le bouc qui était coincé trouva que l'eau montait. Une planche en bois intervint et il sauta dessus, le puits déborda et il sorti. »; l'enseignant avait indiqué «mal dit » sur la copie).

Des déterminations d'un autre ordre peuvent également fonder de façon concomitante ou bien de façon indépendante le sentiment de justesse, à savoir des considérations de nature stylistique. Les normes que j'ai recensées entrent, en effet, en relation avec d'autres normes qui forment une sorte de toile de fond dans le maillage de l'évaluation lexicale. Les normes de l'emploi ou du réinvestissement, de l'appropriation et celle de la précision ou spécificité lexicale prennent sens en partie en référence à quatre 
normes stylistiques, qui définissent des principes ou règles d'écriture identifiant un bien écrire : la norme de la richesse et de la variété lexicale, celle de l'évitement des répétitions et de l'emploi surveillé du registre familier. Les deux premières, qui se superposent parfois, font figure de normes "mères", des normes syncrétiques, qui surplombent les autres. Sur le plan conceptuel, elles représentent une condition de la connaissance et de l'emploi du "mot juste". Marie-Anne Paveau et Laurence Rosier (2008) considèrent que la richesse, entendue au sens de quantité et de qualité, représente l'un des stéréotypes les plus courants sur le lexique et constitue un argument dans les jugements concernant la qualité de la langue. Les trois enseignants des classes observées attendent en littérature dans les récits de fiction de leurs élèves l'emploi de verbes de parole variés; dans deux classes, la norme en tant que critère de l'évaluation a été communiquée oralement lors du rappel des consignes.

Les deux autres normes partenaires de l'idée de "mot juste", qui sont elles aussi des normes qualitatives porteuses d'une axiologie, font figure de normes propres à l'évaluation des réalisations lexicales. L'évitement des répétitions - que rend possible la connaissance de "mots justes" - a été présenté par deux des trois enseignants comme un critère d'évaluation constant en littérature ; le critère (code « $R »$ ) figure pour l'une des classes dans la grille d'auto-évaluation permettant à l'élève, sa production une fois corrigée par l'enseignant, de comptabiliser ses erreurs par catégorie et pour l'autre classe dans la grille de relecture ("Il n'y a pas de répétition ») utilisée par l'élève avant la "mise au propre". La norme de l'évitement du registre familier, autrement dit d'un emploi réduit et conditionné du registre, est elle aussi une norme stylistique qui peut être mise en relation avec l'idée de "mot juste" et qui fait partie d'une sorte de système conceptuel sur lequel l'idée est susceptible d'apporter un éclairage : un mot relevant d'un registre dit familier n'est pas reconnu la plupart du temps comme un "mot juste" à l'inverse d'un mot relevant d'un registre identifié comme soutenu ou bien courant. La norme dans deux des classes observées a été indiquée par l'enseignant lors des entretiens sachant qu'une tolérance s'applique aux séquences de paroles rapportées directement («mais dans un dialogue ça passe ce genre de choses »). En effet, seuls quelques placements de mots ou groupes de mots entre guillemets dans un corps de productions (par exemple l'expression faire sa fête à quelqu'un) laissent deviner la présence de la norme et il est à remarquer que les mots "familiers" sont rares dans les 128 productions collectées. L'un des deux enseignants a expliqué que l'item "vocabulaire » figurant dans la grille d'évaluation accompagnant chaque expression écrite signifie "un niveau de langage» et qu'il fait office de rappel pour les élèves ("une idée de réflexe») incitant ces derniers à user d'un vocabulaire "qui montre de quoi ils sont capables si possible dans toutes les disciplines ».

La présence des registres de langue au chapitre des normes, outre de montrer la présence effective et opératoire de l'idée de "mot juste" en contexte d'enseignement du vocabulaire, souligne donc les liens que l'analyse peut tisser entre la question des normes de l'évaluation et celle des objets d'enseignement. Certaines familles d'objets représentent en effet un lieu d'émergence de l'idée.

\subsubsection{Les objets d'enseignement}

L'idée d'après les sources considérées noue des affinités privilégiées pour des raisons en partie différentes sur lesquelles je m'attarderai avec trois familles d'objets : les mots étudiés et/ou abordés, les registres de langue et les lexiques ou vocabulaires des disciplines. Autrement dit de façon constante, donc commune aux trois familles, et de façon unique dans le cas de la première famille, c'est le caractère d'objet d'enseignement - un enseignement identifié et explicite - associé éventuellement à une attente d'emploi en production qui assigne le statut de "mot juste". Nous l'avons vu à propos des verbes de parole. C'est en ce sens que l'on peut dire que les logiques didactiques afférentes aux objets d'enseignement instaurent un enseignement du "mot juste".

La justesse dans le cas des registres de langue se double d'une question d'usages. La notion de registre, qui renvoie à un type de variation dite diaphasique et parfois dite situationnelle ou stylistique, tente de rendre compte d'usages c'est-à-dire décrit des formes linguistiques dont des formes lexicales et définit les paramètres et règles qui peuvent régir les relations interpersonnelles. La multiplicité, la diversité ainsi que l'hétérogénéité des paramètres, tels que la situation, l'objet, les partenaires, les enjeux de l'échange 
verbal, rendent complexe et délicate la description du type de variation dont relève la notion de registre, d'autant plus que les usages étudiés mettent en œuvre des déterminations d'ordre social, discursif et idéologique notamment et que la notion de registre engage des considérations appréciatives (Gadet, 2007). En contexte d'enseignement du vocabulaire, la connaissances des registres, des registres dits courant, soutenu et familier en particulier, ainsi que l'emploi approprié de ces registres selon les situations de communication pratiquées à l'école, autrement dit les règles d'emploi des registres qui impliquent l'emploi encouragé des registres courant et soutenu et l'emploi surveillé c'est-à-dire circonstancié du registre familier obéissent à une axiologie (un "bon usage") et suivent une inflexion normative. Philippe Perrenoud (1988) rappelle que l'école est dans la société un des lieux qui légifère sur la langue et l'un des lieux où s'exerce un contrôle de la langue qui est enseignée et évaluée ainsi qu'un contrôle des pratiques langagières. Selon Perrenoud (1988: 79), des normes codifiées qui donnent lieu à des attentes et motivent des jugements, des normes qui ne sont pas toujours explicites ou même conscientes, font que l'école exerce une politique normative : "On juge le style et le niveau de langue, l'étendue, la précision ou la distinction du vocabulaire, la correction, l'élégance ou la complexité des tournures ». La connaissance et l'emploi des registres participent d'une norme de langue, les variétés de français scolaire qui définissent la langue à enseigner. Sont enseignés un savoir-parler et un savoir-être. Et, c'est la langue écrite qui est prise pour norme de référence tandis que d'autres formes de la langue, orales par exemple, ordinaires ou populaires sont dévaluées (Gadet, 2007). Un des trois enseignants a déclaré de façon significative lors d'un entretien que la production d'un dialogue engendre "un glissement vers un registre qui n'est pas satisfaisant », "un espèce d'oral à la fois pas construit et pas riche au niveau du sens et au niveau de l'écriture ».

Concernant le dernier objet d'enseignement que j'ai répertorié, les lexiques ou vocabulaires des disciplines, le sentiment de justesse puise en dominante à d'autres sources. Dans les bornes de et en référence à un domaine identifié de connaissance, tel que ce domaine est pensé, constitué et abordé en contexte scolaire, le sens des mots est appréhendé relativement à un référent/des référents daté(s) ou situé(s) historiquement (aussi y-t-il a un sentiment de relative unicité sémantique) et dans ses relations à des connaissances de nature encyclopédique. Un des enseignants a dit au moment d'un entretien à propos de la production d'un élève que la première des deux compétences à acquérir en histoire consiste à «acquérir un certain bagage lexical adéquat approprié à la période historique étudiée». Les connaissances lexicales ressortissent d'ailleurs elles-mêmes dans une certaine mesure à un savoir disciplinaire. Ce sont ces différents traits qui alimentent sur un plan conceptuel un possible sentiment de justesse. Le poids décisif du domaine d'expérience, par conséquent du point de vue adopté et du type de discours tenu, rapproche toute proportion gardée les lexiques ou vocabulaires des disciplines des lexiques ou vocabulaires de spécialités.

L'idée de "mot juste" est donc active relativement à des dimensions et des composantes didactiques nodales. Parce qu'elle siège au cœur de logiques profondes, elle peut être considérée comme représentant une ligne de force. En permettant en outre de mettre en lumière des liens entre des conceptions didactiques qui concernent la question des finalités, de l'évaluation et des objets d'enseignement, elle offre la possibilité d'ébaucher un premier réseau conceptuel. Ces conceptions que nous pourrions comparer à des conceptions de surface, des conceptions de premier niveau, s'articulent à des conceptions d'arrière-plan, des conceptions en seconde épaisseur, qui forment l'épistémologie didactique de l'idée.

\section{L'épistémologie didactique de l'idée de "mot juste"}

Les conceptions dont je considère qu'elles constituent un arrière-plan des dynamiques didactiques liées à l'idée de "mot juste" se rapportent d'une part aux problématiques de la langue et du langage, à celles d'autre part de l'enseignement et de l'apprentissage. Chaque catégorie de conceptions a trait, en effet, à une aire spécifique et apporte un éclairage différent sur des objets d'étude qui dans la recherche en didactique sont foncièrement multidimensionnels. 


\subsection{Les conceptions relatives à la langue et au langage}

Les deux conceptions dégagées sont étroitement liées, l'une formant le pendant de l'autre.

La conception relative au lexique, qui au sein de la philosophie de l'idée de "mot juste" représente une pièce maîtresse, a trait au statut de signe du mot rattachant l'idée en contexte d'enseignement comme en d'autres contextes à la dialectique des mots et des choses, en d'autres termes aux relations langue/monde. Le mot relativement à son potentiel de signification est conçu comme un "outil" ou moyen permettant de référer à des objets ou entités extralinguistiques de divers statuts, permettant donc de désigner et de nommer ces objets ou entités, de les viser. La part dénotative du sens d'un mot, qu'elle soit directe ou indirecte (dans le cas des unités lexicales qui telles les adjectifs et les verbes prédiquent quelque chose d'une autre entité), est prise en compte de façon privilégiée et saillante. Cette conception du mot se place dans une perspective référentielle et "réaliste" et peut envisager le mot comme une étiquette et la langue comme une nomenclature. La conception tend en effet à appréhender la relation référentielle davantage sur le mode du reflet (une relation de correspondance) que sur celui de la conceptualisation (une relation de médiatisation) qui implique une relative autonomie des signes vis-à-vis de l'univers extralinguistique ainsi qu'un ordre propre de ces derniers dans la visée référentielle. Dans les données considérées, la conception se fait jour par deux truchements en particulier. En premier lieu, des annotations portées sur des copies par l'un des trois enseignants projettent en filigrane la conception. J'ai retenu ces deux exemples qui témoignent du caractère directeur des considérations sémantico-référentielles dans leurs relations à des composantes de l'univers fictionnel créé (situations et actions des personnages) : «non, il ne bégaie pas » (l'extrait est: «-Ok, bégaya le bouc». »); «il [le bouc] est dans le puits » (« Une heure plus tards notre bouc rencontra Mr Trémousse avec sa bande d'hacolytes »). Les remarques sousentendent que l'assise forte du sentiment de justesse est de nature sémantique, ce dont j'ai donné un aperçu lors de l'examen des définissants de la justesse. En second lieu, la présence exclusive de verbes et de noms dans les corpus de mots travaillés en histoire et en littérature, la présence par conséquent de deux catégories grammaticales qui relèvent avant tout d'un ordre de signification lexicale, peut au-delà d'un jeu de circonstances être considérée elle aussi comme étant révélatrice sur un plan conceptuel. Rappelons, en outre, que le sens des mots relevant des vocabulaires ou lexiques des disciplines est appréhendé dans ses relations à des référents datés. Les élèves qui devaient rédiger l'article de gazette avaient reçu pour instruction de ne pas commettre d'anachronisme.

La conception, qui voit dans le mot un "outil" ou moyen de référer à des objets ou des entités extralinguistiques et qui accorde de ce fait une place de choix à la dimension dénotative du sens, est solidaire sur le versant du langage d'une autre conception: le langage est pensé comme un moyen d'évoquer et de parler du monde, assurant une fonction de référenciation et de représentation. Le cadre de pensée plus général qui est celui de l'idée de "mot juste" s'enroule et se déploie, en effet, autour d'une logique communicationnelle : l'expression de contenus - ou selon d'autres formulations l'expression d'un sens, l'expression d'un vouloir dire, d'une intention de communication ou d'une intention de signifier constitue le fer de lance de possibles raisons d'être ou motivations à l'emploi du "mot juste". Il me semble que l'un des professeurs a souligné cette logique d'une élaboration et d'une communication d'une signification en mettant en relation des emplois lexicaux avec la réalité psychologique prêtée au personnage de Robinson, ce en liaison avec la situation fictionnelle construite (« il [le personnage] se montre menaçant »), à propos du passage de production suivant :

«-« Que faite vous sur mon île! gronda Robinson.

-« Ben moi et mon équipage nous nous sommes échoués. expliquai-je.

- Et bien vous avez intéraient à dégager de mon île, ou je vous plombe vos fesses. Je vous laisse une semaine, pas plus. Compris? répondit Robinson. » (...)

- Il ne vous reste plus que quatre jours, vous avez intéraient à vous dépécher si vous ne vouley pas danser la lambada! lança Robinson. ».

L'invocation d'une finalité d'intercompréhension, finalité que le motif de la clarté peut également signifier, fait elle aussi partie des motivations possibles à l'emploi du/d'un "mot juste". Aussi l'idée de 
"mot juste" sur fond de conception relative au langage engage-t-elle la question de la relation pensée/langage qui peut être considérée comme un cadre épistémologique englobant, que la relation soit conçue sur le mode de la dualité (l'emploi du "mot juste" se définissant alors comme la traduction exacte ou adéquate de la pensée - la représentation ou le reflet d'un contenu) ou sur celui de la cohésion (l'emploi se définissant cette fois comme l'émergence exacte ou adéquate de la pensée - l'émergence et la formation d'un contenu c'est-à-dire du sens).

Les conceptions qui peuvent être mises au jour présentent par conséquent des ramifications qui dessinent des ensembles conceptuels permettant de saisir des fondements de l'idée de "mot juste" d'un point de vue épistémologique. L'objet ainsi que le champ d'étude qui sont les miens appellent l'emprunt d'angles de vue autres qui sont en jeu dans le contexte qui nous intéresse, à savoir ceux de l'enseignement et de l'apprentissage.

\subsection{Les conceptions relatives à l'enseignement et à l'apprentissage}

Je déplierai trois conceptions, qui entrent en contact et s'enchaînent. Les conceptions de l'apport et de l'emploi renvoient toutes deux à une logique d'enseignement et d'apprentissage si bien que je ne pratiquerai pas de différenciation entre les deux points de vue. La troisième conception, une conception du transfert, qui s'arrime aux deux autres, correspond quant à elle à une conception de l'apprentissage.

La conception de l'apport est chronologiquement première : il s'agit en effet de faire connaître à l'élève des mots qui font figure de "mots justes" en puissance et/ou d'assurer une compréhension sémantique de ces mots. Dans le cas de l'histoire, c'est-à-dire d'un enseignement lexical identifié et balisé par des contenus encyclopédiques, la conception se superpose à une autre conception : celle d'un enseignement et d'un apprentissage en situation. Des modalités d'enseignement témoignent de la conception de l'apport qui peut être considérée comme se dessinant également en creux dans la finalité d'emploi des mots et dans la norme qui lui correspond. Parmi les deux modalités isolées, la liste de "mots justes" a une valeur emblématique. Dans les classes observées, l'élève doit en histoire employer dans sa synthèse ou bien dans la réponse développée qu'il apporte à la question posée des mots et locutions déterminés. Pour ce qui est de la production en littérature, il peut dans une des classes consulter la liste de verbes de parole qui sert de référentiel et avait dans les deux autres à réviser avant l'évaluation la liste constituée. Ces listes qui se présentent comme des aides et des guides à l'écriture - une sorte de prêt-à-écrire - définissent une forme d'enseignement qui postule elle-même une forme d'apprentissage : l'apport. C'est en ce sens que je considère que la conception renvoie à une logique d'enseignement et d'apprentissage. Une seconde modalité dans les séquences en littérature met en œuvre la conception : les exercices choisis, qui relèvent du principe du texte à trous (compléter des phrases ou bien des textes ne comportant pas de verbes de parole, placer dans un texte les verbes d'une liste donnée) ou reposent sur des substitutions (remplacer le verbe dire par un verbe plus "précis") et appellent par conséquent l'emploi du ou d'un "mot juste", mettent en pratique un apport qui a déjà eu lieu ou qu'ils réalisent. La conception renferme donc sur le versant de l'apprentissage l'idée que ce dernier passe par une exposition et/ou une imprégnation.

Les racines, qui participent de l'assise représentationnelle de la conception de l'emploi, ne sont pas radicalement différentes. La conception qui voit dans l'emploi des mots une modalité d'enseignement et une modalité d'apprentissage du "mot juste" fait de l'emploi une forme d'exposition et d'imprégnation. Cette seconde conception semble constituer sur le plan didactique un tandem avec la conception de l'apport. La fonction des exercices que j'ai référencés, à savoir une pratique, met en exergue la conception. Pour ce qui concerne les séquences en histoire, le fait que la production demandée représente à la fois un vecteur et une étape de l'apprentissage atteste la conception. L'enseignement explicite en littérature et en histoire d'un vocabulaire identifié, la finalité générale d'emploi ou de réinvestissement ainsi que la norme d'évaluation afférente à la finalité figurent les autres points d'attache de la conception, qui paraît par conséquent être dotée parce qu'elle se fixe sur différentes composantes didactiques d'une certaine valeur opératoire et partant d'une certaine représentativité.

Il appert en aller de même pour ce qui concerne une conception du transfert. Cette troisième conception présente la particularité d'être couplée aux deux autres. Les conceptions de l'apport et de l'emploi 
présupposent en effet une logique de transfert. En termes d'apprentissage, la conception de l'apport mise sur un transfert, celle de l'emploi sollicite un transfert.

Les cinq conceptions mises en avant contribuent donc à éclairer des fondements épistémologiques de l'idée de "mot juste". Or, les conceptions dégagées ne sont pas seulement significatives de l'extension de l'idée en contexte d'enseignement du vocabulaire, mais le sont aussi de la portée de celle-ci. Une lecture critique des conceptions épistémologiques dans les relations qu'elles entretiennent avec les points d'émergence de l'idée montrera en effet en quoi l'idée de "mot juste" peut être tenue pour un révélateur.

\section{L’idée de "mot juste" : un révélateur}

L'idée fait en effet ressortir des orientations didactiques qui à la fois paraissent majeures en enseignement du vocabulaire et sont le creuset d'aspects problématiques.

\subsection{Un révélateur d'orientations saillantes}

L'orientation qui semble surplomber les autres est celle de l'enrichissement lexical. Les différents points d'ancrage de l'idée de "mot juste", en particulier les normes de l'évaluation, ainsi que la conception de l'apport se rattachent d'une façon plus ou moins ample et plus ou moins directe à une finalité d'enrichissement, faisant de celle-ci un centre de gravité de l'enseignement du vocabulaire. L'enrichissement lexical vise un emploi et autant que possible un emploi approprié des mots. Ce que recouvre l'idée parce qu'il prend sens par rapport à une préoccupation d'enrichissement souligne donc l'importance ainsi que l'extension de la préoccupation en tant qu'axe structurant. La finalité d'enrichissement entre en relation avec une représentation, dont un des trois enseignants a fait état explicitement, la pauvreté ( $e$ en fonction du niveau socioculturel l'élève est doté d'un bagage lexical riche ou pauvre »; "il y en a qui ont un vocabulaire très pauvre»). La pauvreté lexicale prêtée aux élèves ou à certains élèves forme en quelque sorte l'image inversée, presque converse, d'une autre représentation tout aussi globale et floue, la richesse lexicale dont j'ai mentionné une actualisation - la norme stylistique de la richesse. La représentation de la pauvreté, qui procède d'une perception connotée et négative, raisonne en termes de manque ou de carence, alliée à celle de la richesse, serait l'expression d'une sorte de cadre de pensée bipolaire fondateur dans lequel s'inscrirait l'idée de "mot juste". L'idée remplit d'autant plus un rôle de révélateur qu'elle aide à approcher la nature de l'enrichissement et d'une certaine manière une facette de la pauvreté.

Ce qui se rattache à l'idée met en effet en relief la place centrale qu'occupe la question du sens. La préoccupation d'enrichissement, c'est-à-dire d'extension du répertoire lexical des élèves, paraît faire la part belle à la dimension sémantique, qui va de pair avec une dimension formelle (la connaissance de la forme phonologique et orthographique du mot) et une dimension catégorielle (la connaissance de la classe grammaticale d'appartenance du mot). Rappelons que j'ai évoqué la primauté des considérations de nature sémantique lors de l'examen des normes de l'appropriation et de la précision ou spécificité lexicale. Plusieurs autres des données concourent au même constat. La plupart des mots et locutions travaillés en histoire, dont un certain nombre figurent dans la liste qui définit un cadre et guide une restitution dirigée de connaissances encyclopédiques, ont été définis à l'oral et/ou à l'écrit. Le sens ou un élément de sens a lui aussi été indiqué, le plus souvent à l'oral, au sujet d'un grand nombre des verbes de parole travaillés ou bien rencontrés lors d'activités ou d'exercices. Les verbes figurant dans la liste qui sert de référentiel aux élèves sont classés selon un critère d'ordre sémantico-énonciatif ; par exemple, dans l'une des deux classes concernées : expression d'un ordre/d'un conseil, d'une interrogation, d'une déclaration, d'une exclamation. Nombre d'exercices, enfin, qui dans deux classes ont pour but de faire employer des verbes de parole donnés trahissent eux aussi de par leur type - des exercices fonctionnant sur le principe du texte à trous, des exercices consistant à remplacer le verbe dire par un verbe plus "précis" - la primauté de considérations sémantiques. L'idée de "mot juste" tend donc à mettre en avant la place décisive qu'occupe la dimension sémantique dans l'enseignement lexical. 
Cette place minore et laisse plus ou moins dans l'ombre d'autres dimensions qui jouent pourtant un rôle dans l'emploi des mots. Aussi les deux orientations que je viens de présenter posent-elles pour une part question.

\subsection{Un point de cristallisation de dimensions problématiques}

L'emploi des mots, un enseignement qui repose en partie sur des implicites et se situe peu en conséquence dans une logique d'apprentissage constituent les deux pôles problématiques que j'aborderai.

La dimension sémantique dans le domaine de l'emploi des mots représente, il est vrai, une dimension déterminante. Cependant, d'autres dimensions entrent en jeu au point que la problématique de l'emploi des mots gagne à être envisagée comme configuration mobile de multiples dimensions. Des déterminations d'ordre sémantique, syntagmatique, discursif et textuel notamment se conjoignent et se combinent selon un jeu de saillances qui selon toute vraisemblance peut varier en fonction des situations discursives et des mots, sachant que les déterminations d'ordre sémantique forment l'axe névralgique et structurant d'une sorte d'"alchimie" lexicale. L'analyse de passages qui dans des productions d'élèves peuvent être considérés comme étant significatifs engage à soutenir un tel point de vue. Le choix par exemple d'un titre pour l'article de gazette («Grand drame à la cour», "Catastrophe à la cour du roi ») dénote de la part d'élèves l'alliance de données discursives relatives à la fonction de l'écrit produit ("accrocher" l'attention de lecteurs potentiels et susciter l'envie d'en savoir davantage) à des aspects sémantiques (le sens en contexte des mots grand, drame et catastrophe). Dans une autre classe, toujours en histoire, l'emploi par exemple du substantif ancêtre ("La manufacture était l'ancêtre de l'usine ») peut lui aussi être lu comme marquant le point de rencontre de considérations sémantiques et discursives : l'emploi du substantif sied en effet à un écrit scolaire de type informatif en dominante, la synthèse, qui implique l'adoption d'une orientation didactique (la tenue d'un propos vulgarisant). Du côté des productions en littérature, les trois extraits suivants donnent un aperçu de l'intrication de considérations d'ordre sémantique et textuel avant tout. Des emplois lexicaux réalisés peuvent être mis en relation avec des faits de littérarité, en l'occurrence des traits génériques (la figure littéraire de Renard dans les fables ; les thématiques de la ruse et de la naïveté ; un scénario de duperie) :

«Une fois que Renards fût au dehor, il pencha au dessus du puits et s'écria :

"Que tu es niais mon ami aux grandes cornes, crois-tu vraiment que j'ai l'intention de te sortir de là? »;

«-Trève de bavardage, bon... ah oui, mets toi contre le mur je grimperait le premier le long de ton échine! le bouc s'exécuta. ».

Relativement à la situation fictionnelle relatée (un retournement qui suit un scénario de vengeance/revanche et marque le triomphe de Bouc sur Renard), des emplois peuvent être mis en relation avec la forme de parole rapportée adoptée (successivement une parole rapportée directement puis une parole rapportée indirectement) :

«Capitaine Renard se plaignit que se n'était qu'une farce. Mais Sire Bouc n'en écouta pas un mot. Celui-ci se mit à foncer dans Renard les cornes droites devant. Renard qui n'était plus qu'une galette se sentit plaqué contre le mur et le poid de Bouc sur son dos.

«On fais moins le malin », se moqua Bouc et il partit. ».

Ces différents emplois lexicaux instituent un réseau de significations et créent des effets de sens.

Or, rien d'après les annotations portées, les critères retenus, les déclarations faites lors des entretiens dont les informations fournies à propos des grandes lignes des séquences conduites, ne porte trace d'une prise en compte franche et différenciée d'aspects discursifs et/ou textuels dans leurs relations aux emplois lexicaux. En effet, soit les aspects sont absents, soit ils affleurent mais demeurent confus car ils appartiennent à un point de vue global sur la production. C'est ainsi qu'un des enseignants au vu de ses déclarations semble ignorer des paramètres d'ordre syntaxique et discursif lorsqu'il ne se montre sensible 
qu'à des paramètres d'ordre sémantique en commentant le caractère inapproprié de l'emploi du verbe papoter («-«Voilà on à fini, entrons à la maison »

\section{-D'accord allons y » lui ai-je papoté»).}

L'emploi du verbe ne convient pas pour des raisons également syntaxiques (*papoter à quelqu'un) et textuelles (la place du verbe en position conclusive et non ouvrante pour introduire une séquence de paroles rapportées directement). La référence faite, en outre, par un seul enseignant en situation d'entretien (l'explicitation de la mise entre guillemets de la locution faire sa fête à quelqu'un: "c'est familier le bouc parle comme ça c'est d'une efficacité redoutable ») à des considérations textuelles, ce de façon ponctuelle et globalisée, ne peut être tenue pour suffisamment significative d'une prise en compte nette et régulière, voire constante. Le quasi-silence qui entoure de possibles variables d'ordre syntagmatique, discursif et/ou textuel ainsi que la position de retrait qui semble prévaloir dans l'enseignement d'aspects syntagmatiques - propriétés syntaxiques et propriétés de cooccurrence des mots - interrogent l'enseignement du vocabulaire en ce qui concerne l'emploi des mots. Par voie de conséquence, ils interrogent en amont la finalité d'enrichissement qui est une finalité générale et de ce fait une finalité floue et au final contre-productive ainsi que la prédominance accordée à la composante sémantique. Celle-ci, en effet, n'est pas autonome mais est corrélée, voire se nourrit de d'autres composantes. Cette prédominance aurait d'autant plus besoin d'être interrogée que l'abord du sens des mots (une dimension dénotative du sens, la recherche d'invariants sémantiques - des éléments de sens stables -) semble privilégier un point de vue en langue et non en discours.

La primauté de la dimension sémantique qui rejette en arrière-plan des dimensions syntagmatique, discursive et textuelle notamment tend à rendre implicites l'enseignement ainsi que l'apprentissage de ces dimensions. Or, cette situation pose elle aussi question. Les conceptions de l'apport, de l'emploi et du transfert, d'autre part, qui font en partie reposer l'enseignement et l'apprentissage sur une part d'implicite, étant donné de surcroît que des dimensions extra-sémantiques ne semblent pas véritablement prises en compte ni mises en lumière, ne raisonnent pas ou peu en termes d'appropriation de variables intervenant dans l'emploi des mots. C'est à l'élève au cours des différentes activités proposées par l'enseignant (en histoire, lecture/analyse des documents support de la leçon, leçon elle-même; en littérature, lecture d'extraits d'œuvres de littérature de jeunesse, exercices de relevé et d'emploi des verbes de parole), autrement dit à partir d'exemples, par conséquent de situations et de contextes d'emploi, de discerner et de construire par lui-même des règles d'emploi. La logique dominante suivie est donc plus une logique d'enseignement qu'une logique d'apprentissage. À cet égard, la liste des "mots justes" à employer (des mots "nus") s'avère peut-être emblématique de l'hégémonie d'une logique d'enseignement. Or, il y a un pas de la connaissance ou d'une connaissance du "mot juste" à l'emploi du "mot juste". Dans une étude consacrée à l'enseignement du vocabulaire, Hubert Dupart (2002:49) considère lui aussi que l'emploi des mots pose question et constate dans l'état des lieux qu'il dresse de pratiques d'enseignement au cycle 3 notamment que les activités proposées s'appliquent plutôt à mettre en place un savoir sur les mots et non à faire usage des mots ni à faire entrer ces usages dans des productions langagières, si bien que le moment venu, celui de la production de texte par exemple, l'élève doit opérer un «bond qualitatif» et "passer d'un savoir sur le mot à un savoir se servir du mot, d'un savoir sur à un savoir employer (qui installe un savoir sûr). ». Il est, en outre, légitime de se demander dans quelle mesure les annotations portées par l'enseignant pour ce qui est des productions en littérature, si ce dernier ne les explicite pas auprès de l'élève ou s'il ne propose pas de solution, voire n'aide pas l'élève à en trouver une, peuvent contribuer à une formation effective et constructive à l'emploi des mots et à des principes qui régissent l'emploi. Les annotations - soulignement, mise entre guillemets ; «mal dit», « mal choisi »; «formule autrement » (maladresse d'expression) ; «verbe de discours 》 - ne sont pas en effet forcément éclairantes ni limpides pour l'élève. Des difficultés potentielles liées à une part d'implicite dans l'enseignement et à une mise en sourdine d'une problématique d'appropriation se cumulent. 


\section{Conclusion}

L'idée de "mot juste" me semble donc pouvoir être considérée comme une clé de compréhension possible c'est-à-dire comme une clé d'exploration et de questionnement. Ce qui se rapporte à l'idée en contexte d'enseignement du vocabulaire permet, en effet, de mettre au jour et de mettre en lumière des dynamiques ou des tendances didactiques majeures ainsi que des conceptions épistémologiques qui leur sont associées et de pointer par là même des dimensions problématiques. Aussi l'idée peut-elle être comparée à un cristallisateur. Elle renferme des paradoxes : par exemple, affirmer une finalité d'emploi, attendre une appropriation ou bien une précision ou spécificité lexicale, et en contrepartie ne pas problématiser ni clarifier auprès de l'élève ce qui peut être en jeu dans l'emploi des mots. Les différentes analyses que j'ai menées tendent à montrer qu'un enseignement du "mot juste" - un "mot juste" potentiel et un enseignement de nature sémantique - l'emporterait sur un enseignement de l'emploi du "mot juste" - un enseignement de paramètres déterminant l'emploi des mots, ce qui suppose un enseignement de plusieurs propriétés des mots -, l'un des postulats étant que le premier renfermerait le second, en d'autres termes que la connaissance du sens d'un mot en particulier suffirait à son emploi. Une problématique d'enrichissement resterait la plus forte.

La question de l'emploi des mots par conséquent demeure en didactique du vocabulaire un domaine ouvert qui appelle à la réflexion et commanderait un enseignement plus explicite, plus ample et plus complet, en résumé un enseignement plus structuré et plus structurant. L'optique de justesse lexicale intéresse cependant des faits délicats et complexes, car elle convoque et se penche sur des aspects sémantiques, syntaxiques, discursifs et textuels notamment sans oublier des aspects qui ont trait aux usages, liés eux-mêmes aux contextes d'utilisation des mots et aux discours afférents.

\section{Références bibliographiques}

Authier-Revuz, J. (1995). Ces mots qui ne vont pas de soi Boucles réflexives et non-cö̈ncidences du dire. Tome 2. Larousse, collection Sciences du langage.

Dupart, H. (2002). Apprendre le vocabulaire Choisir une pédagogie. Lyon : Chronique sociale, Pédagogie formation l'essentiel.

Gadet, F. (2007). La variation sociale en français. Ophrys, collection L'essentiel français.

Paveau, M-A. \& Rosier, L. (2008). La langue française passions et polémiques. Paris : Vuibert.

Perrenoud, P. (1988). Chapitre IV. La langue française est-elle gouvernable?. Neufchatel-Paris : Delachaux et Niestle.

Pottier, B. (1992). Sémantique générale. Paris : PUF, Linguistique nouvelle. 